\title{
Optimization of Computer Network Teaching and Learning Behavior Using Virtual Experiment Technology
}

\author{
Xiaohua Li, Renlong Zhang*, Li Yang, Quanyue Yang, Juan Nie \\ School of Computer and Information Engineering, Beijing University of Agriculture, \\ Beijing, China
}

\begin{abstract}
Teaching and learning behavior in classroom is an important factor that affects the quality of classroom teaching. It is the basic conditions to achieve curriculum philosophy. In this paper, we firstly analyze the main problems existing in the current teaching of computer network, and then we present our study and practice on the optimization of computer network teaching and learning behavior. We apply virtual experiment technology into computer network classroom teaching. We pay attention to the creation of learning environment using virtual experiment technology, and we emphasize students and teachers analyze and explore in the scenarios. Through discussion, competition and cooperation, students achieve their efficient learning experience and construct their own knowledge. In this way, we combine theory and practice during the teaching process, thus optimize the classroom teaching behavior. Practical results show the learning process is feasible and efficient.
\end{abstract}

Keywords: Teaching and learning behavior; Constructivism; Virtual experiment technology; GNS

\section{Introduction}

Teaching and learning behavior in classroom is an important factor that affects the quality of classroom teaching. It is the basic conditions to achieve curriculum philosophy. In accordance with the syllabus requirements of the computer network course, students should obtain the following achievements:

- Understand the development process of computer networks.

- Understand the basic concepts and principles of computer network.

- Grasp the theory and technology of the computer network system.

However, the reality may be far below the requirements. Typically, after completing their studies, the students may still be short of perceptual knowledge on computer network, or they just stay on rote memorization. In view of this, we firstly analyze the main problems existing in the current teaching of computer network in this paper. Then, we present our study and practice on the optimization of computer network teaching and learning behavior.

\section{Problems existing in the current teaching of computer network}

Computer Network not only contains abstract and profound theoretical knowledge such as the principles of network communication, network communication protocols and algorithms, but also involves a large number of 
network technologies such as operation and configuration of networking equipment. The problems existing in the teaching of computer network are as follows:

- Lack of flexibility in teaching methods. The traditional "forcedfeeding" teaching model is still in wide use. In this unidirectional teaching, the teacher just spoonfeeds the lessons and students learn by rote, they do not like to speak out or ask questions in class, their initiative and enthusiasm are not mobilized.

- Heavy theory, light practice. There is a common phenomenon in the teaching of Computer Network in quite a number of universities: they pay less attention to the practice with too much importance attached to the theory teaching. But they ignored this fact that this course is both theoretical and practical, pure theory teaching is not feasible. It is necessary to perform theory verification and analysis in practice.

- Lack of laboratory equipment. The computer network course involves a great amount of network technology and network equipment. To study these contents, only verbal explanations and illustrations are not feasible. We must possess a certain amount of experimental facility for theory verification and analysis. As network technology develops so rapidly and the replacement speed of the related equipment is so fast, most universities cannot provide adequate equipment for theoretical classroom teaching due to funds or other reasons, not to mention they provide laboratory equipment to students for self-study or self-test after class.

- Single form of evaluation methods.
At present, close examination is still the main way of examination. Relying on mechanical memorizing, students will be able to cope with the examination. The examination results cannot reflect the actual conditions of students, cannot accurately evaluate teaching effect, and cannot be used to guide the teaching.

All these make it difficult to achieve ideal teaching effectiveness with traditional teaching method. Inspired by the core idea of Constructivism [1-5], we apply virtual experiment technology into the computer network teaching to optimize the teaching behavior.

\section{Virtual experiment technology}

Virtual experiment means with the help of multimedia, virtual reality technology, people can simulate the operational aspects of the traditional experimental on computers. With the help of the mouse, keyboard and other equipment, by clicking or dragging and dropping and other movements, the students realize the virtual operation of equipment, to complete the virtual experimental experience.

Compared with the traditional physical experiments, virtual experiments are low cost and they don't consume equipment. Moreover, people can carry out virtual experiments of any scale, without having to worry about the limitation of the amount of equipment. On the other hand, virtual experiments are fast, high efficient. Generally, there is a wide range of network equipment and other devices in the virtual experiment software package, people can use it to do various experiments conveniently.

GNS (Graphical Network Simulator)[6] is a very good graphical virtual network experiment platform that can simulate the real IOS and complex networks. It is an 
excellent complementary tool to real labs for network engineers, administrators etc.

\section{Teaching behavior optimization us- ing virtual experiment technology}

Classroom teaching behavior is the key to the implementation of the curriculum, and any curriculum ideas should be realized in the classroom teaching behavior. To optimize computer network teaching and learning behavior in the classroom teaching, we pay attention to the creation of realistic learning environment using virtual experiment technology. In terms of computer network course, we have created quite a lot of learning scenarios with GNS. In the scenarios, students and teachers are driven by a series of tasks. To accomplish the tasks, they analyze, explore in the ocean of knowledge. They share the teaching and learning experience with each other. Through discussion, competition and cooperation, students achieve their efficient learning experience and construct their own knowledge. Take RIPv2 routing authentication knowledge point for example, one of the learning scenarios we create with GNS is shown as Fig. 1. In order to analyze the safety performance of the two authentication modes, we use Wireshark to capture the RIPv2 packets sent by the routers. Unpacking these packets captured, the students can easily understand the PDU formats of the two authentication mode and master the implementation mechanisms. They can compare RIPv1 and RIPv2 Message Formats, and feel the difference between classful routing protocols and classless routing protocols in this scenario. After unpacking these packets the student also can come to such conclusions: MD5 encrypted authentication is safer than plain text authentication; MD5 encrypted authentication can effectively prevent eavesdropping on the line to obtain the authentication key phenomenon.

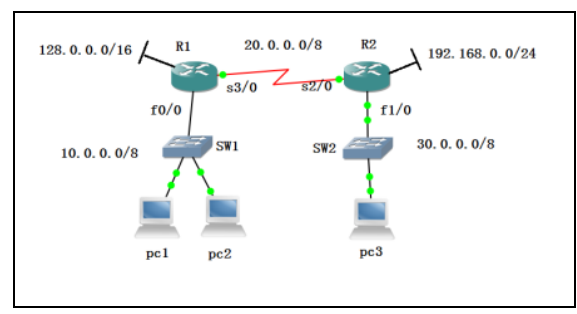

Fig. 1: RIPv2 routing authentication learning scenario

To consolidate the knowledge points, teacher and students should summarize and make a review. Another point to note is that they should also carry out teaching evaluations. Evaluations can be carried out from the following three aspects: independent learning ability, contributions made during the learning process, whether the meaning construction is complete. Through evaluation, the dominant position of students learning is fully embodied, students' enthusiasm and initiative are inspired as well.

The last step of our optimizations is to provide a more complex learning scenario, called improving practice. Thus, students can carry on a deeper exploration. Back to the RIPv2 routing authentication knowledge point example, the scenario we provided is shown as Fig. 2.

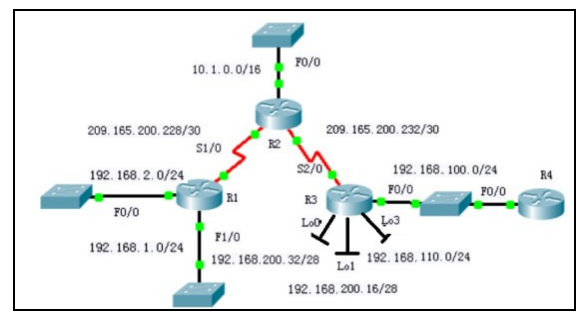

Fig. 2: RIPv2 routing authentication improving practice

In this way of learning, the students not only obtain deeper understanding of the problem, but also get an intuitive feel for 
real practicing skills. Abstract theory becomes concrete, vivid and easy to understand and master. The knowledge points that originally appear to be scattered and messy are easily interspersed together by the exploring main line. Unconsciously, the students' meaning constructions of knowledge are completed.

\section{Conclusions}

In this paper, we present our study and practice on the optimization of computer network teaching and learning behavior. The optimization using virtual experiment technology to create vivid learning scenarios, it emphasizes studentcentered and pays attention to fully mobilize students' learning initiative and enthusiasm. Practical teaching results indicate the learning process is feasible and efficient.

\section{Acknowledgements}

This work is sponsored by the project of Beijing Committee of Education (KM201210020004). Renlong Zhang is the communication author. We thank the authors involved in our reference for sharing beneficial inspirations with us. We also thank the peer review experts for their guidance and advice. Many references had to be deleted due to limited space, and we apologize to authors and readers for work that could not be cited.

\section{References}

[1] L. S. Hu and P. Li, "On computer teaching based on constructivism theory," in 2011 International Conference on Sport Material, Modelling and Simulation, ICSMMS 2011, January 27, 2011 - January 28, 2011, Shenzhen, China, pp. 560-564,(2011).

[2] H. Li, "Constructivism-based multimodal teaching model of college english writing," in 2011 3rd Pacific-Asia Conference on Circuits, Communications and System, PACCS 2011, July 17, 2011 - July 18, 2011, Wuhan, China, pp.1-4,( 2011).

[3] Z.-Y. Liu, Y.-H. Jiang, G. Chen, S.-G. $\mathrm{Wu}, \mathrm{R}$. Chen, and C.-Z. Guan, "Application of constructivism theory explore electronic technology design course teaching," in International Conference on Advances in Computer Science, Environment, Ecoinformatics, and Education, CSEE 2011, August 21, 2011 - August 22, 2011, Wuhan, China, pp. 299-302,(2011).

[4] J. Pan, M. Zhang, T. H, and L. T, "The physics network teaching system design and implementation based on constructivism," in 2nd Annual Conference on Electrical and Control Engineering, ICECE 2011, September 16, 2011 - September 18, 2011, Yichang, China, pp. 6973-6976,(2011).

[5] H. Yahong, W. Weihong, and J. Li, "Teaching discrete mathematics with the constructivism learning theory," in 6th International Conference on Computer Science and Education, ICCSE 2011, August 3, 2011 - August 5, 2011, Singapore, Singapore, pp. 815-816,(2011).

[6] GNS, "http://www.gns3.net/." 